\title{
Creating a transatlantic research enterprise for preventing Alzheimer's disease
}

\author{
Zaven S. Khachaturian ${ }^{a,{ }^{*}}$ [Convener \& Co-Chair], Jordi Camíb [Co-Chair], Sandrine \\ Andrieu $^{\mathrm{c}}$, Jesús Avila ${ }^{\mathrm{d}}$, Mercè Boada Rovira ${ }^{\mathrm{e}}$, Monique Breteler ${ }^{\mathrm{f}}$, Lutz Froelich ${ }^{\mathrm{g}}$, Serge \\ Gauthier $^{h}$, Teresa Gómez-Isla ${ }^{\mathrm{i}}$, Ara S. Khachaturian ${ }^{\mathrm{a}}$, Lewis H. Kulleri, Eric B. Larson ${ }^{\mathrm{k}}$, \\ Oscar L. Lopez', José Manuel Martinez-Lage', Ronald C. Petersen ${ }^{\mathrm{m}}$, Gerard D. \\ Schellenberg $^{\mathrm{n}}$, Jordi Sunyer ${ }^{\mathrm{o}}$, Bruno Vellas ${ }^{\mathrm{p}}$, and Lisa J. Bain ${ }^{\mathrm{q}}$ [reporter]
}

aLou Ruvo Brain Institute, Las Vegas, NV, USA bFundació Pasqual Maragall I Parc de Recerca Biomédica de Barcelona (PRBB), Barcelona, Spain ${ }^{\circ}$ Department of Internal Medicine and Clinical Gerontology, Acute Unit for Alzheimer's Patients, Toulouse, France ${ }^{\mathrm{d} C e n t r o ~ d e ~ B i o l o g i ́ a ~}$ Molecular Severo Ochoa (CSIC-UAM), C/ Nicolás Cabrera, 1 Universidad Autónoma de Madrid, Cantoblanco, Madrid, Spain eDirección Médica de Fundació ACE, Instituto Català de Neurociències Aplicades, Barcelona, Spain fDepartment of Epidemiology and Biostatistics, Erasmus Medical Center, Rotterdam, The Netherlands 9Division of Geriatric Psychiatry, Central

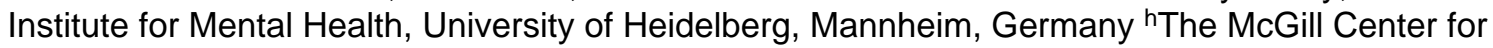
Studies in Aging, McGill University, Montreal, Quebec, Canada 'Department of Neurology, University of Navarre, Pamplona, Spain iDepartment of Epidemiology, University of Pittsburgh, Pittsburgh, PA, USA kGroup Health Center for Health Studies, Seattle, WA, USA 'Department of Neurology, University of Pittsburgh School of Medicine, Pittsburgh, PA, USA mDepartment of Neurology, Mayo Clinic, Rochester, MN, USA nDepartment of Pathology and Laboratory Medicine, University of Pennsylvania School of Medicine, Philadelphia, PA, USA ${ }^{\circ}$ Centre for Research in Environmental Epidemiology, Barcelona, Catalonia, Spain PDepartment of Geriatrics, INSERM U558, Toulouse University Hospital Center, Toulouse, France 9Science and Medical Writer, Elverson, PA, USA

\section{Abstract \\ In recognition of the global problem posed by Alzheimer's disease and other dementias, an international think-tank meeting was convened by Biocat, the Pasqual Maragall Foundation, and the Lou Ruvo Brain Institute in February 2009. The meeting initiated the planning of a European Union-North American collaborative research enterprise to expedite the delay and ultimate prevention of dementing disorders. The key aim is to build parallel and complementary research infrastructure that will support international standardization and inter-operability among researchers in both continents. The meeting identified major challenges, opportunities for research resources and support, integration with ongoing efforts, and identification of key domains to influence the design and administration of the enterprise.}

\section{Introduction}

Over the coming decades, Alzheimer's disease (AD) and other dementias will become the number one public-health problem worldwide, exceeding an astonishing 100 million people by 2050 [1]. Recognizing that a worldwide problem of this scope demands worldwide 
solutions, AD experts from Europe and North America met in Barcelona, Spain, in February 2009 to begin planning a European Union-North American (EU-NA) collaborative research enterprise, tentatively called the North Atlantic Successful Aging Union (NASAU), aimed at developing effective interventions to slow the progression and ultimately prevent the disease. This think-tank meeting was organized by Biocat the BioRegion of Catalonia in collaboration with the Pasqual Maragall Foundation (Barcelona, Spain) and the Lou Ruvo Brain Institute $^{1}$ (Las Vegas, NV). The EU-NA partnership is only a first step. Eventually, researchers from other parts of the world will be invited to join a worldwide consortium of scientists and political leaders working together, sharing data, and putting the resources of all countries together to find solutions for the problem of AD. This goal will not be achieved in 5 or 10 years; the ambition is to identify solutions by 2020. Participants at the meeting were tasked with "thinking big," on the order of the Human Genome Project, as they deliberated about ongoing programs, initiatives, and existing resources on which to build this collaboration.

The Barcelona meeting was conceived as a planning workshop to begin identifying existing programs, initiatives, and resources that can be built upon and leveraged to meet the overarching goals of the EU-NA collaborative program:

- To create the infrastructure needed to conduct early detection and prevention studies by identifying early risk factors, biomarkers, genetic markers, and other data that will allow for predictions of an individual's likelihood of developing dementia.

- To provide resources that researchers can use to test hypotheses or generate new hypotheses related to causation and intervention. These resources include both new instruments that will need to be developed and validated, and large, diverse cohorts of well-characterized individuals to participate in trials of new instruments and interventions.

- To use these resources and infrastructure in the development of interventions that will delay, ameliorate, or change the course of $\mathrm{AD}$, or prevent the disease altogether.

- To attract more young investigators to the field by making these resources broadly available and by providing access to the leading scientists in the field from around the world.

\section{Defining the challenge}

The meeting began with an overview of the problem, which was characterized as "the tsunami of healthcare, worse even than the economic crisis we are now seeing." The incidence of dementia is rising astronomically because of our aging population. In 2002, the United Nations Population Division reported that the number of people aged 60 years or older tripled over the previous 50 years, and will triple again over the next 50 years. Couple that with the association between increased age and the incidence of dementia: the incidence of dementia doubles approximately every 5 to 6 years after age 65 [2], meaning that among individuals 80 to 85 years old, as many as $40 \%$ to $45 \%$ have some form of dementia. More importantly, research over the past 30 years shows that by the time a person is diagnosed with dementia, the disease has already caused neurodegeneration of vast areas of the brain. Hippocampal volume loss, one of the earliest measurable signs of $\mathrm{AD}$, quantifies damage that has already occurred. Yet we do not have tools to measure important antecedent conditions such as dendritic and synaptic loss. Such measures may one day be used as

\footnotetext{
${ }^{1}$ Now renamed Cleveland Clinic Lou Ruvo Center for Brain Health
} 
biomarkers of $\mathrm{AD}$ to identify neurodegeneration earlier and diagnose the disease in its early stages, but equally important, without these tools, we cannot even begin to understand the fundamental mechanisms that underlie dementia: why synapses are being lost, and why dendrites are pruned in specific ways. Yet such an understanding is critical if we are to develop effective preventive strategies.

Among the universe of people who will develop dementia, there is a wide range of patterns and trajectories in which the condition will progress. After the disease process begins, cognitive function remains at a plateau for a variable amount of time, and then begins to decline at variable rates. The goal of preventive therapy is thus to increase the length of the plateau, and to slow the slope of decline. From a diagnostic and treatment perspective, the challenge is to predict who among normal individuals will likely develop incident dementia at younger ages, i.e., less than 65 years, and at older ages, 285 years. Such information can lead to strategies for preventing the development of key risk factors (i.e., primordial prevention), and therapies to slow the progression to clinical dementia. This will require the application of existing technologies (e.g., imaging, biomarkers, and genetic risk factors) to younger people, and the development of new technologies, new genetic markers, and new biomarkers. The overall goal is to extract as much information as possible from very early indicators, to predict an individual's disease trajectory and how a putative treatment might alter that trajectory. The multifactorial nature of the disease will undoubtedly require a pattern analysis of various indicators, as has been the case in genomics and proteomics studies. Eventually, we hope to identify the relevant set of indices so that when an intervention becomes available, appropriate individuals can be selected to test the effectiveness of that intervention.

With existing technologies and the lack of a validated surrogate marker for $\mathrm{AD}$, prevention trials take 15 to 20 years before results can be available. Realistically, this must be shortened to about 3 to 5 years, which will require new technologies and surrogate markers. To convince pharmaceutical regulatory bodies such as the Food and Drug Administration and European Medicines Agency (EMEA) to accept new technologies and surrogate markers, they will have to be validated on large, diverse populations. Thus the need for a diverse population cohort is twofold: first, to validate new technologies and biological markers that can serve as proxies (i.e., surrogate markers) for the biological process that leads to dysfunction; and second, to identify individuals in the earliest stages of the disease, for enrollment in short-term clinical trials of preventive strategies.

\section{Building on previous studies}

Previous large population studies offered many lessons, and possibly the foundation on which to build the EU-NA research enterprise. Hence part of the strategy in creating a planning team was to invite investigators who had participated in many of these studies. Based on their experiences with previous and ongoing studies, these investigators from Spain, France, The Netherlands, Germany, Canada, and the United States are in an ideal position to consider whether existing cohorts can be adapted for new, worldwide studies. For example, since the original cohorts were enrolled, new analytical methods and new biomarker tests have become available that could be added to ongoing studies. In addition, because most of these studies were built on the cardiovascular model, they may not have included tests of cognition. Another potential drawback with existing cohorts involves the age of enrolled participants. Insofar as many of these studies focused on an aging population, they may not have enrolled younger subjects who would be the focus of a study looking at early indicators of dysfunction. However, in some cases, existing cohorts can be expanded to include younger participants. Previous studies that were discussed at the Barcelona meeting included: 
- The Rotterdam Study, a longitudinal study of chronic disease including cognitive impairment, in subjects aged over 55 years. The study began in 1990 and was subsequently expanded to include subjects as young as age 45 years. The cohort now includes 15,000 subjects, about 10,000 of whom are still living. In 1995, an imaging component was added in a subcohort of participants.

- Alzheimer's Disease Neuroimaging Initiative (ADNI) and Euro-ADNI are comprehensive efforts to identify neuroimaging and other biomarkers of mild cognitive impairment (MCI) and AD. The ADNI investigators have made a major effort to standardize imaging protocols, so that data can be collected at multiple sites. The ADNI expects to enroll 800 participants between ages 55 and 90 years: 400 with MCI, 200 with early AD, and 200 controls. A subset of participants will also undergo analysis of amyloid plaque burden by positron-emission tomography scanning with Pittsburgh Compound B.

- The Cardiovascular Health Study (CHS) began in 1989 as a longitudinal (10-year) study of risk factors for the development of heart disease and stroke in people aged $\checkmark 65$ years. Originally, 5201 participants were enrolled at four locations, and another 687 (primarily African Americans) were added in 1992. Cognitive function was also assessed in participants, and the CHS Cognition Study was initiated in 1992, adding magnetic resonance imaging to the protocol.

- In France, the Multidomain Alzheimer Preventive Trial, conducted by the Centre Hospitalier Universitaire de Toulouse, is underway in Toulouse, Bordeaux, Limoges, and Montpellier, and will include 1200 frail, elderly volunteers during a 3 -year period. Nearly 400 participants have been recruited. This study will assess the impact of omega-3 fatty acid and of a multidomain intervention program on cognitive decline.

- The German Competence Network on Dementias has enrolled 3327 subjects aged $\geq 75$ years without initial cognitive decline, who are being followed by their general practitioners at six different urban sites linked to university research centers or memory clinics. Ongoing follow-up has reached the 3-year mark. In another cohort, 2113 patients with early signs of AD or MCI plus controls were followed for 2 years at 12 academic memory clinics. Extensive neuropsychological and clinical data, structural magnetic resonance imaging, DNA, and cerebrospinal fluid were collected in a biomaterial bank.

- In Canada, 33 clinics form a clinical trial network named The Consortium of Canadian Centres for Clinical Cognitive Research (C5R). A Sarkozy-like initiative is planned in Quebec Province.

- In Spain, investigators are studying the effects of environmental exposure on neural development. In particular, they have evidence that mercury may have some impact, but because it can act in combination with other factors, they are looking at the data geographically. This is a 4-year study enrolling 4000 children, in whom the investigators are seeking early markers of neural development and metabolic syndrome. They are also interested in air pollution and potential effects on cognition through the mechanism of oxidative stress or neuroinflammation. One problem involves the difficulty of measuring exposure.

- The Genome-Wide Association Studies are a National Institutes of Health (NIH) initiative to identify genetic factors involved in various conditions, including AD. The Alzheimer's Disease Genetic Consortium is running gene arrays on samples of well-characterized cohorts from various studies, including the ADNI cohort. They expect to have data on 2000 cases and 2000 controls. 
- The European Community Concerted Action on the Epidemiology and Prevention of Dementia Group was a collaborative effort in the early 1990s that brought together population-based cohorts underway at that time in Europe. They established many connections and collaborations among investigators, which may be useful in designing the EU-NA collaboration.

A number of important lessons were gleaned from the examination of these studies, and will be considered in shaping the EU-NA enterprise. Many studies of aging collected biological samples and data relevant to cognitive health (e.g., cardiovascular health), without including specific cognitive measures. Adding cognitive measures to these studies would be a relatively easy way of extending the scope of these studies, and the participants at the Barcelona meeting agreed on a position statement strongly recommending that measures of cognition or brain dysfunction be included in all population studies related to aging, given that the number one cause of morbidity and mortality is rapidly becoming dementia as a result of increased longevity. It is extremely important that high-quality measures of cognition and risk of dementia, including brain imaging, be included in all clinical trials of older individuals that evaluate potential risk factors for dementia, including blood pressure, diabetes, exercise, lipids, inflammation, and diet. Moreover, the potential benefits and adverse effects of new drug therapies that will be given to large numbers of older individuals should evaluate the effects of such drugs on cognition and ultimately on the risk of dementia.

In addition, it became clear that different cultures and healthcare delivery systems in participating countries shaped previous studies, and will add complexity and challenges to new studies and consortia. But perhaps the predominant lesson from reviewing these studies is that much of the foundation already exists upon which to build the EU-NA enterprise.

\section{Laying the groundwork: Who, what, when, where, and how?}

Participants at the Barcelona meeting began a discussion of the broad areas to be addressed by the consortium. Elaboration of these focus areas will continue through the efforts of working groups and subsequent group meetings.

\subsection{Cohort: Who?}

The goals of this research enterprise demand that the study cohort reflect different cultures, genetic backgrounds, and access to services. This will require standardization across many centers around the world. Participants should also be available for serial measurements. Given the large number of existing cohorts assembled for the above-mentioned studies and others, participants questioned whether a new cohort is needed, or if existing cohorts can be combined, concatenated, or expanded. Another question concerned whether a subgroup of people who have aged successfully should be included, to study factors that contribute to healthy brain aging.

\subsection{Domains: What?}

In determining what to measure, a number of governing principles emerged. First was the need to remain flexible, because both our technology and our understanding of the biological basis of $\mathrm{AD}$ are rapidly evolving. Mechanisms for adapting to new knowledge and technology will have to be built into any study design. The second principle states that brain aging cannot be disentangled from aging in other physiologic systems, such as the kidney and heart. Worldwide, there will be a tremendous explosion of aging people in Asia, Latin America, and Africa, where people have different health problems than in the developed world. Therefore, we will need to understand brain function against the backdrop of other health changes. The third principle maintains that the amount of information from 
various measures and the amount of accompanying "noise" are not constant. Thus, a measure that provides much information at later stages of the disease may not give much information at early stages (e.g., certain imaging studies). Moreover, the relationship between biological and clinical phenotypes is not clear. Thus to extract information, measurements will be required across multiple domains, such that investigators can look for patterns of change. Individual change in one domain may not be very useful by itself, but in the context of other changes, it can become meaningful. It will be imperative to determine how changes relate to one another.

Keeping these basic principles in mind, the group identified a number of domains that will be important to measure. Some disagreement arose about the relative importance of measuring changes in the brain versus changes in function.

Cognition, of course, is the key domain, and within this domain, memory, attention, executive function, speed of processing, language, and visuospatial performance are all important. In a typical person with $\mathrm{AD}$, memory problems become apparent first, followed by problems with executive function and language. Embedded within the area of memory, measures of short-term versus consolidated memory may be needed. Several issues were discussed involving the cognitive domain. Some participants expressed concern about a ceiling effect with cognitive measures. As the population becomes increasingly educated, it becomes more difficult to detect differences, especially in early stages of the disease. An ideal cognitive battery would contain an entry level (different at different ages), as well as a threshold where everyone fails in some aspect. Whatever measures are used, they will have to be designed so that they are neither education-dependent nor culture-dependent.

Other domains of the study include an assessment of mood and behavior, which can be affected up to 10 years before dementia is detected. Activities of daily living are recognized as a key metric that divides MCI from AD. However, no standardized measures are available for the predementia stages of AD. Motor function can be assessed using the timedwalk, up-and-go, and two-step treadmill tests. To supplement tests of motor function, we may want to include assessments of muscle mass and strength. In the cardiovascular area, at minimum, blood pressure should be measured. Other measures to consider in this area include arterial stiffness and pulse-wave velocity. In the metabolic domain, we should include body mass index and food-frequency questionnaires. It will also be important to collect data on family history. In the area of genetics, we will want to collect data regarding the question of what genetics can tell us about the early stages of the disease. Expression profiling may allow an assessment of patterns of genetic changes, and these may relate to other health problems.

The study will also involve the collection of biological materials, including blood and cerebrospinal fluid, and possibly neurological tissue for environmental studies. Serum samples would be used not only for biomarker studies, but also to evaluate the overall health of other organ systems through laboratory tests such as hemoglobin A1c (HbA1c), creatinine, and glucose. DNA from blood would be used to develop new genetic markers and evaluate existing genetic risk factors.

Another key component of the study will involve imaging. For this, the ADNI study can be used as a template, although it does not presently examine vascular dementia. The ADNI protocol was designed to focus on the conversion from MCI to AD, and from normal function to MCI. We would like to look more broadly at antecedents for cognitive dysfunction. Thus, although ADNI and European-ADNI have made significant progress in developing systematic protocols, we may need new technologies for imaging early changes in the brain, including new ligands for positron-emission tomography. Other poorly 
understood areas where imaging may provide useful tools include neurogenesis in the dentate gyrus, and synaptic and dendritic pruning.

\subsection{Data gathering: How and when?}

A primary goal of this enterprise is to make data-gathering a routine part of the general health examination, similar to the way blood-pressure and cholesterol measurements are performed presently. Cognitive assessment should also be included as a part of general medical practice, so that data are collected in a systematic way, and sampled in a standard way. General practitioners, or primary-care providers, will be especially important on the front line of the data-gathering effort. Thus protocols should be structured as simply as possible, to collect a representative sample. A user-friendly toolbox for general practitioners is essential. This may include computer-based or web-based assessments that can be set up at a kiosk, although an alternative instrument may be needed for rural or other areas that lack broadband internet access, or where computers are not available. Self-administered assessment tools could also be considered.

In designing data-gathering tools, it will be important to bear in mind the overall burden on participants and clinicians (particularly if primary-care providers are involved), because too heavy a burden could decrease compliance with the study. One possible strategy would involve a basic "toolkit" that collects a minimal amount of data from everyone, and then focuses on certain areas at specialized centers. Another issue relating to both participant and clinician burden pertains to the collection of bodily fluids. The collection and storage of samples will need to be standardized and monitored. One suggestion advocated an "optimal" standard as well as an "acceptable" standard, with samples coded so that investigators would know which samples are usable in certain studies. For example, for proteomics studies, sample collection needs to be tightly controlled.

The frequency of data collection from each participant will also need to be determined. The data-collection visits may increase as a participant ages.

\subsection{Data management}

Managing data presents another set of questions to be resolved. For example, should data be stored in a centralized location, or at multiple, decentralized locations? Should blood and tissue be stored in a central repository? Once again, looking to existing studies should provide some guidance in answering these questions. A minimal, international data set will be established, to ensure that comparisons can be made across sites.

Data sharing, data mining, and knowledge extraction emerged as possible barriers that will require creative solutions. The NIH require data from NIH-funded studies to be shared through qualified-access databases, but some European countries will not allow their scientists to share data. One possible solution would permit members of the consortium special exemptions to share data across international boundaries. Decisions will also have to be made regarding who will have access to data. A number of possible models exist. The ADNI, for example, provides access to almost everyone, and what people do with the data is unrestricted. However, this has resulted in some misinterpretation and misuse of data, as well as conflicting results from different investigators analyzing the same data. In the Genome-Wide Association Studies, everyone who contributes data can take their own data, but not aggregate data, back to their home site. Other studies restrict access to data, or provide only portions of the data to investigators outside the study.

The issues of data sharing and standardization need to be resolved at the outset. Convoluted mechanisms for sharing data interfere with scientific progress, and this has not been successfully addressed in existing or previous international collaborations. In Europe, 
databases with clinical data and biomaterials are difficult to share outside the researchers' home country. Moreover, when attempting to share data between countries, laws and ethical standards and issues of ownership, intellectual property, and privacy all require careful consideration and management.

\subsection{Sites: Where?}

Existing large cohort studies may provide the easiest and quickest path to start NASAU (i.e., the EU-NA enterprise). Potential United States study sites that may be invited to participate include the Mayo Clinic and the University of Pittsburgh Alzheimer Disease Research Center (ADRC), the Pittsburgh Collaboration with Barcelona, the Women's Health Initiative (multiple sites), the CHS (multiple sites), the Action to Control Cardiovascular Risk in Diabetics (ACCORD) study trial (multiple sites), and the Framingham Heart Study (Boston, MA). Potential sites in Canada include those in the C5R network.

In Europe, possible sites include the German network, the French Center for Excellence in London, Spain's clinical trial network (the Consorcios Asociados de Investigación Biomédica en Red), the Million Women Study in the United Kingdom, and the Monica project in France (Paris, Toulouse, and Strasbourg). Collaborating with the Sarkozy Initiative in France may also be possible.

\subsection{Administration}

As currently conceived, the EU-NA enterprise would involve joint programming and two parallel structures with overlapping leadership, to facilitate cross-talk between the EU and NA investigators. After we have demonstrated the practicality of this model through our working group of scientists, the NIH, the Canadian Institutes of Health Research, and the European Commission may be convinced to involve themselves and support the project. Presently, two cross-Atlantic models are being developed. One, a collaboration initiated between Bruno Vellas in France and the late Leon Thal's Alzheimer's Disease Cooperative Study (ADCS) in San Diego, continues to design a multidomain trial regarding prevention. The second model is the Pittsburgh-Barcelona collaboration. We expect to build on and expand these models.

The ADNI provides another model that may serve as a template for designing the administrative aspects of this study. Similar to the German Competence Network, the ADNI has a central administrative core with an external advisory board, an industry scientific advisory board, a resource-allocation committee, and centralized facilities for data banking, data mining, data storage, and several technology cores, such as the neuroimaging core at the University of California at Los Angeles. The ADNI has taken advantage of the ADCS for clinical coordination, and also has a data-coordinating center. Genetic data are stored at one site, which offers advantages in terms of distribution: more sophisticated users can obtain raw data, whereas other users can access data in other, more usable forms.

Informational technology administration can be particularly difficult to manage across countries and continents because of the different technologies that are used. This is another area to be addressed.

\section{Moving forward}

This initial meeting of the EU-NA Research Enterprise on the Prevention of Dementia identified the long-term goals of the collaboration and the necessary steps that must be taken to achieve these goals. To this end, a number of working groups will be established to continue planning efforts. One group will work on solidifying the domains to be studied, on the additional types of data and biological samples that will be needed, and on the conditions 
for collecting these samples. Another workgroup will evaluate computer-based measures. A further workgroup will analyze existing databases, combing the literature more systematically and cataloguing the designs and executions of existing studies, to determine how we might be able to piggyback on them. Workgroups will also be established to plan the administrative structure, a systematic public education effort, and funding strategies.

Benefits of the EU-N A enterprise could extend well beyond the central mission of developing the resources to perform large studies on diverse populations. International collaborations can be tremendously attractive to young investigators seeking to establish themselves in a fast-moving research area. In addition, by establishing an exclusive "club" of investigators doing high-quality work, the enterprise may enhance participants' success in receiving funding from the NIH, the Canadian Institutes of Health Research, the European Commission, and other agencies. High-quality data may also encourage these agencies to develop funding mechanisms that would use the resources of the consortium.

This collaboration is conceived as being inclusive, and in the coming months, additional investigators will be invited to join the working groups and help shape the enterprise.

\section{References}

1. Brookmeyer R, Johnson E, Ziegler-Graham K, Arrighi HM. Forecasting the global burden of Alzheimer's disease. Alzheimers Dement. 2007; 3:186-191. [PubMed: 19595937]

2. Ziegler-Graham K, Brookmeyer R, Johnson E, Arrighi HM. 2008. Worldwide variation in the doubling time of Alzheimer's disease incidence rates. Alzheimers Dement. 2008; 4:316-323. [PubMed: 18790458] 